\title{
PRÉSENCE DANS LE LAIT ET LA GLANDE MAMMAIRE DE COMPOSÉS GLUCIDIQUES LIÉS AU GUANOSINE 5' DIPHOSPHATE
}

\author{
R. DENAMUR, G. FAUCONNEAU et Geneviève JARRIGE-GUN'TZ \\ Station de Recherches de Physiologie animale, \\ Centre National de Recherches Zoolechniques, Jouy-en-Josas.
}

CABIB et LELOIR ont isolé en I954, à partir de la levure, un dérivé glucidique du Guanosine $5^{\prime}$ diphosphate, (GDP $5^{\prime}$ ), le guanosine diphosphate mannose (GDPM). La présence de ce nucléotide fut ensuite signalée dans différents tissus, secrétions et organismes: oviducte de poule (STROMINGER, I955), Penicillium chrysogenum (BALLIO et a1., I956), laits de brebis, de chèvre, de truie, glandes mammaires de brebis (DENAMUR et al., I958 $a$-b - I959 $a$-b), Evemothecium ashbyii (Pontis et al., I959).

Le guanosine diphosphate fucose (GDPF) est un autre dérivé glucidique du guanosine $5^{\prime}$ diphosphate, récemment identifié à partir des laits de brebis, de chèvre, de truie, de la glande mammaire de brebis (Denamur et a1., I958 $a-b-$ I959 $a-b$ ), ainsi que d'Aerobacter aerogenes (GINSBURG 1958).

Parmi les glucides liés au GDP $5^{\prime}$ le glucose et le mannose sont quantitativement les plus importants, cepandant d'autres dérivés de la guanosine ont été signalés. Ainsi, nous avons décelé la présence de glucose et de galactose dans les sommets de GDP M et de GDP F, résultant d'un fractionnement à froid sur Dowex I, de la fraction acidosoluble des laits de brebis (Denamur et al., I958). PonTrs et al. (I959) ont fait une observation analogue à partir d'Eremothecium ashbyii, où 1'hydrolyse du GDPM libéré en même temps que le mannose une certaine quantité de glucose et de fructuose (la somme glucose-fructose est égale à 6 p. Ioo du mannose). Ces derniers auteurs sont conduits à admettre l'existence de GDP glucose et de GDP fructose. Par ailleurs Poncrs (I957) a isolé à partir de la levure un composé dérivé du GMP $3^{\prime}$ qu'il n'a pu identifier, et HEA'Tr le GDP $5^{\prime}$ colitose à partir d'Escherichia Coli.

Nous avons poursuivi l'étude des fractions glucidiques qui paraissent liées au GDP $5^{\prime}$ dans les laits de brebis, de chèvre et de truie, ainsi que dans les glandes mammaires de brebis prélevées à des périodes différentes de l'activité sécrétoire (gestation, lactation).

L'analyse des nucléotides acidosolubles des laits et de la glande mammaire a été pratiquée selon la méthode de HurLBERT et al. (I954), mais à tue température 
comprise entre o et $2^{\circ} \mathrm{C} *$, ce qui nous a permis d'isoler dès le fractionnement primaire, le GDP M et le GDP F en deux sommets distincts, $\mathrm{H}$ et $\mathrm{H}^{\prime}$ (voir figure).

Afin de rechercher si ces sommets renferment des dérivés glucidiques du GDP 5 , différents du GDP M et du GDP F, les nucléotides des sommets $\mathrm{H}$ et $\mathrm{H}^{\prime}$ sont débarrassés de l'acide formique par extractions répétées à l'éther, puis adsorbés sur charbon (élimination des sucres phosphates) et élués par le mélange éthanol-ammoniaqueeau $(50 \mathrm{~V}-49 \mathrm{~V}-\mathrm{IV})$. Les éluats sont alors chromatographiés en éthanol-acétate d'ammoniaque $\mathrm{M}(75 \mathrm{~V}-30 \mathrm{~V}-\mathrm{pH} 7,5)$ selon la méthode de PALAdini et LeIorr

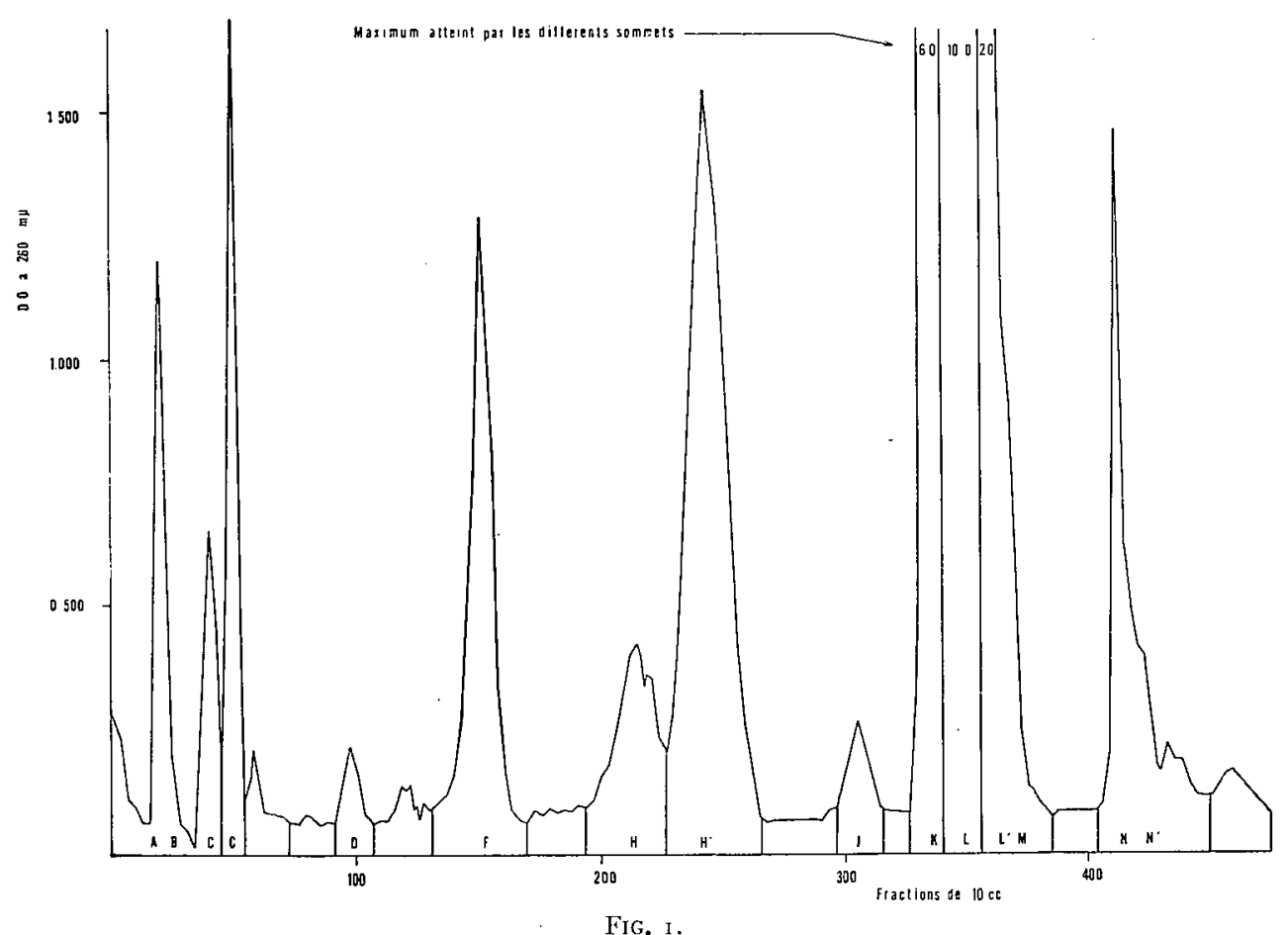

Nucléolides acido-solubles du lait de truie ( $100 \mathrm{ml}$ de lait)

Fractionnement obtenu sur colonne de Dowex $13,2 \times 40 \mathrm{~cm}$ à l'aide d'un gradient acicle torinique $(0-5 \mathrm{~N})$. Formate d'anmonium $(0-\mathrm{I}, 6 \mathrm{~N})$.

(r952), ou soumis à un deuxième fractionnement sur colonne de Dowex I, à l'aide d'un gradient formiate d'ammonium. Aptès migration sur papier, les "spots " correspondant au GDP $\mathrm{M}$ et au GDP F sont élıés à l'eau. Après chromatographie secondaire sur colonne, les sommets de GDP M et de GDP F sont séparés du formiate d'ammonium par adsorption sur charbon puis élution en milieu alcalin. L'analyse des substances ainsi isolées a permis l'identification de la fraction nucléotidique et la mise en évidence de plusieurs composés glucidiques liés à cette fraction.

a) Le G $\mathrm{DP}_{5}{ }^{\prime}$ représente le seule constituant nucléotidique ainsi que les analyses suivantes le démontrent :

\footnotetext{
* Tous les fractionnements chromatographiques (japier ou colomne) adsorptions sur charbon et élutions, ainsi que les élutions des papiers chromatographiques sont effectuées à o-2 ${ }^{\circ}$ en raison de la labilité du GDP $I^{\prime}$.
} 
- Les spectres U. V. à pH I - 7- I3, sont caractéristiques de la guanosine;

- Le GDP $5^{\prime}$ et le GMP $5^{\prime}$ sont seuls mis en évidence après hydrolyse $\mathrm{HCl}$ o, I N. La séparation chromatographique de ces composés a été réalisée à la fois par migration sur papier en éthanol-acétate d'ammoniaque $\mathrm{M}(75 \mathrm{~V}-30 \mathrm{~V}-\mathrm{pH} 7,5)$ et par fractionnement sur colonne de Dowex $\mathbf{x}$.

- La guanine est la seule base identifiéeaprès hydrolyse par l'acide perchlorique, $7^{2} \mathrm{p}$. I0o à $100^{\circ} \mathrm{C}$ pendant $\mathrm{I}$ heure et chromatographie en isopropanol-acide chlorhydrique concentré-eau $\left(678 \mathrm{~V}-\mathrm{r} 76 \mathrm{~V}-\mathrm{I}_{4} 6 \mathrm{~V}\right)$.

b) Les composés glucidiques ont été isolés à l'aide de différentes méthodes :

Les "spots " ou les sommets secondaires correspondant au GDP M et au GDP F sont hydrolysés par $\mathrm{HCl}\left(\mathrm{pH} 2,30\right.$ minutes, $\left.100^{\circ} \mathrm{C}\right)$. Les hydrolysats débarrassés des nucléotides par échangeurs d'anions sont chromatographiés en plusieurs solvants utilisés pour l'identification des sucres : butanol-acide acétique-eau $(4 \mathrm{~V}-\mathrm{IV}-5 \mathrm{~V})$, pyridine-acétate d'éthyle-eau ( $\mathrm{V}-2 \mathrm{~V}-2 \mathrm{~V}$ ), phénol-ammoniaque 3 p. roo eau-cyanure de potassium, butanol-pyridine-eau ( $3 \mathrm{~V}-\mathrm{I} \mathrm{V}-3 \mathrm{~V})$. L'ionophorèse en tampon borate selon la méthode CONSDEN et al. (I952) ainsi que la chromatoionophorèse (première dimension : phénol-ammoniaque ot pyridine-acétate d'éthyleeau; deuxième dimension : ionophorèse en tampon borate $\mathrm{pH}$ 9) ont également été utilisées.

La similitude du comportement chromatographique et ionophorétique, ainsi que les tests de surcharge, associés à différentes révélations, nous ont permis d'identifier outre le mannose et le fucose, déjà connus, le glucose, le galactose, l'arabinose et plusieurs oligosaccharides. La présence et l'importance relative des divers glucides doivent être en visagées séparément dans la glande mammaire et le lait.

\section{A. - LE LAIT}

Io Les hexoses: le glucose et le galactose ont été observés dans les deux sommets de GDPM et de GDP F du lait de truie, et dans le colostrum, le lait "ocytocique "* et le lait de fin de lactation chez la brebis. Dans tous ces cas, le glucose est en quantité plus importante que le galactose, il existe seul dans le lait de brebis secrété $d u 20^{\circ}$ jour a11 Ig $\mathrm{6}^{\mathrm{e}}$ jour de lactation.

$2^{\circ}$ Les pentoses et autres sucres inconnus: $1^{\prime}$ arabinose a été isolé à partir du GDP F dans le colostrum de brebis. Un autre sucre de Rf (glucose) : I,43 en pyridine - acétate d'éthyle-eau existe dans le GDP M des colostrum de brebis et dans le lait de chèvre. Enfin, un sucre de Rf (glucose) 2,7 (jaune citron après tévélation par la benzidine, acide trichloracétique) a été trouvé dans le colostrum de brebis.

$3^{\circ}$ Les oligosaccharides nous avons constaté la présence de trois oligosaccharides $(a, b, c)$ de Rf (glucose : $0,15,0,40,0,65)$ en pyridine-acétate d'éthyle-eau.

L'oligosaccharide $b$ qui est le plus important quantitativement, présente les mêmes caractéristiques de migration que le lactose dans tous les solvants utilisés et après ionophorèse.

* Le lait " ocytocique " est obtenu de la manière suivante : après la traite normale, on évacue le lait résiduel avec 20 U. I. d'ocytocine synthétique (Syntocinon). 30 minutes après, on pratique une deuxième vidange complète de la glande mammaire avec $20 \mathrm{U}$. I. d'ocytocine. Le lait secrété pendant les $3 \circ$ minutes est appelé lait " ocytocique "il est resté un temps limité dans les Acini mammaires, contrairement au lait normal qui peut subir certaines modifications pendant l'intervalle de iz heures qui sépare habituellement deux traites consécutives. 
Il a pu être isolé en quantités assez importantes à partir des GDP F du lait de truie, du lait ocytocique et du colostrum de brebis. Son hydrolyse par l'acide sulfurique $\mathrm{N}$, (I heure, I00 ${ }^{\circ} \mathrm{C}$ ), conduit à du glucose et du galactose en quantités à peu près équivalentes, à 1'oligosaccharide $c$ et à deux sucres de Rf (glucose) : I,43 et 2,7 en pyridine-acétate d'éthyle-eau.

Les sommets de GDP F du colostrum et du lait ocytocique de brebis, du lait de chèvre et de truie renferment l'oligosaccharide $a$.

L'oligosaccharide $c$ a été isolé à partir des sommets de GDP M et de GDP F du lait de truie.

Les oligosaccharides $a$ et $c$ ont été obtenus en quantités trop faibles pour qu'il ait été possible d'étudier leur composition.

Enfin, nous avons décelé la présence d'oligosaccharide dans le lait de brebis.

\section{B. - LA GLANDE MAMMAIRE}

I $^{0}$ Les hexoses : le glucose et le galactose existent à tous les stades gestatifs étudiés $\left(75^{\mathrm{e}}\right.$ jour $-90^{\mathrm{e}}$ jour $-\mathrm{I} 2 \mathrm{O}^{\mathrm{e}}$ jour $-\mathrm{I} 35^{\mathrm{e}}$ jour $)$ dans les del1x sommets primaires correspondant au GDP M et au GDP F, mais le rapport galactose/glucose est supérieur à $I$. Les deux hexoses ont été également isolés au cours de la lactation ( 4 heures, Io jour, I $5^{\mathrm{e}}$ jour, $54^{\mathrm{e}}$ jour) dans les deux sommets GDP M et GDP F, mais avec une importance relative plus grande pour le glucose. En fin de lactation ( $150^{\mathrm{e}}$ jour, I $8 \mathrm{o}^{\mathrm{e}}$ jour) le galactose n'a pas été détecté, le glucose existe en faibles quantités.

$2^{\circ}$ Les pentoses: 1'arabinose semble localisé dans le sommet de GDP F du fractionnement primaire. Sa présence est observée pendant la gestation (I20 jour, I $35^{\mathrm{e}}$ jour) et au début de la lactation ( 3 heures, 24 heures, $I 5^{\mathrm{e}}$ jour). Les quantités d'arabinose peuvent être très importantes; elles égalent celles du fucose et sont supérieures à celles du glucose et du galactose à la $24^{\mathrm{e}}$ heure de sécrétion.

Un autre pentose de Rf (glucose) I,44 en pyridine-acétate d'éthyle-eau, a été détecté dans le GDP F, en fin de gestation et au $\mathrm{r} 5^{\mathrm{e}}$ jour de lactation.

$3^{\circ}$ Les oligosaccharides : an $\mathrm{I}_{5}{ }^{\mathrm{e}}$ jour de la lactation nous avons observé dans le sommet de GDP F l'existence d'un oligosaccharide migrant comme le lactose dans les 3 solvants utilisés (butanol-pyridine-eau ; pyridine-acétate d'éthyle-eau; butanol acide acétique-eau). Les quantités isolées étaient trop faibles pour permettre l'étude de sa constitution. Nous n'avons pu déceler la présence d'oligosaccharide pendant la gestation.

\section{DISCUSSION}

Différentes techniques ont été utilisées pour isoler et purifier le GDP M et le GDP F : deux fractionnements sur colonne à l'aide de gradients d'élution différents, deux adsorptions sur charbons ou un fractionnement sur colonne auquel s'ajoutent une adsorption sur charbon et une chromatographie sur papier ; aucune ne nous a permis de séparer le glucose, le galactose, l'arabinose et les différents oligosaccharides du mannose et du fucose liés au GDP $5^{\prime}$.

A la suite de ces résultats il semble logique d'admettre que ces glucides sont 
liés at GDP $5^{\prime}$ comme le sont le mannose et le fucose ; ainsi, nos recherches aboutiraient à l'isolement des nucléotides suivants : GDP $5^{\prime}$ glucose, GDP 5 ' arabinose, GDP $5^{\prime}$ galactose, divers GDP $5^{\prime}$ oligosaccharides. Le type de liaison existant entre GDP $5^{\prime}$ et les glucides ne peut être précisé.

Il faut cependant signaler que le glucose et le galactose ont été isolés à partir des deux sommets de GDP M et de GDP F, même si ces derniers sont complètement séparés au cours du fractionnement primaire. Dans ce dernier cas, il est difficilement concevable que le GDP galactose et le GDP glucose soient élués de la colonne à 2 positions différentes. D'autre part, si les oligosaccharides existent surtout dans le GDP F, on en trouve également dans le GDP M. Bien que 1'analyse de la constitution de ces oligosaccharides ne soit qu'ébauchée, l'oligosaccharide $b$ étudié renferme surtout du glucose et du galactose. Il est alors probable qu'une fraction du glucose et du galactose existants dans les sommets de GDP M et de GDP F provient de la dégradation plus ou moins complète d'oligosaccharides liés au GDP $5^{\prime}$.

Des études en cours ont pour but d'éclairer cette question essentielle, que l'on peut poser également pour l'arabinose et les autres sucres inconnus détectés.

Il est prématuré de discuter le rôle métabolique de ces divers composés glucidiques liés au GDP 5' puisqu'aucune réaction de transglycosylation n'a été démontrée pour le GDP M et le GDP F. Par analogie avec le rôle des UDP liés à divers glucides, on peut penser qu'ils interviendraient dans la biosynthèse des glycoprotéines ou des divers polysaccharides du lait (produits finis de sécrétion ou intermédiaires dans. la synthèse du lactose). A cet égard les laits de truie, de vache, de ratte, de femme, contiennent de nombreux oligosaccharides tandis que le lait de brebis, à notre connaissance, n'a pas été étudié.

Reçu en juin 1960.

\section{SUMMARY}

THE OCCURENCE IN MILK AND IN THE MAMMARY GLAND OF GLUCID COMPOUNDS BOUND TO GUANOSINE $5^{\prime}$ DIPHOSPHATE.

Analysis of the acid-soluble nucleotides of milk and of the mammary gland carried out at a temperature around $\mathrm{O}^{\circ} \mathrm{C}$. has enable us to isolate $\mathrm{DPG}$-mannose and $\mathrm{DPG}$-fucose in two distinct peaks $\mathrm{H}$ and $\mathrm{H}^{\prime}$ (see figure).

Different techniques have been used to isolate and purify the DPG-mannose and DPGfucose : two column fractionnations using different elution gradients, two carbon adsorptions or a column fractionnation in conjonction with one carbon adsorption and paper chromatography. None enabled us to separate glucose, galactose, arabinose and different oligosaccharides from the 5 DPG-linked mannose and fucose. It therefore secms logical to admit that these carbohydrates. are linked to $5^{\prime} \mathrm{DPG}$ as are mannose and fucose. The kind of link existing between $5^{\prime} \mathrm{DPG}$ and carbohydrates cannot be specified.

\section{RÉFÉRENCES BIBLIOGRAPHIQUES}

Ballio A., Casinovi C., Serlupi-Crescenzi G., i956. Occurence of free Nucleotides in Penicillium. Chry. sogenum. Biochim. Biophys. acla, 20, 4I 4-4I 5 .

Cabib E., Leloir L. F., I954. Guanosine diphosphate Mannose. J. Biol. Chem., 206, 779-790.

CONSDEN R., STANiter W. M., I952. Ionophoresis of sugars on paper and some applications to the analyses of protein saccharides complex. Nature, $169,783-785$.

Denamur R., Fauconntia G., Guntz G., I958. Isolement d'un nouveau nucléotide dans le lait de. Brebis : Le guanosine 5' diphosphate fucose. 'C. R. Acad. Sci., 246, 2820-2823. 
Denamur R., Fauconneau G., Guntz G., 1958. Les nucléotides liés aux glucides dans le lait. Congrès Int. Biochimie (Vienne).

Denamur R., Fauconneau G., Guntz G., 1959. Les nucléotides acido-solubles des laits de Brebis, Vache, Chèvre et Truie. Rev, esp. Fisiol., 15, 30I-3IO.

Denamur R., Fauconneau G., Guntz G., 1959. Les nucléotides acido-solubles de la glande mammaire de Brebis. C. R. Acad. Sci., 248, $253 \mathrm{I}-2533$.

Ginsburg V., Kirkman H. N., I958. Isolation of guanosine diphosphate fucose from Aerobacter Aevogenes. J. Amer. Chem. Soc., 80, 348I.

HeAtu E. C., Ig6o. The isolation of guanosine diphosphate Colitose from Escherichia Coli. Biochem. Biophys. Acta, 39, 377-378.

Hurlbert R. B., Scinimiz H., Brumm A. F., Potter V. R., i954. Nucleotide Metabolism. II Chromatographic separation of Acido-solubles mucléotides. J. Biol. Chem., 209, 23-37.

Paladini A. C., Leloir L. F., I952. Studies on Uridine diphosphate glucose. Biochem. J. 51, 426-4,30.

Pontis H. G.., I957. A new guanosine nucleotide from brewer's yeast. Biochem. Biophys. Acta, 25, 4I $7^{-}$ 428.

Pontis G. H., JAMES A. L., BADDILEY J., I959. Guanosine diphosphate glucose and guanosine diphosphate fructose in Eremolhecium Ashbyi. Biochem. Biophys. Acta, 33, 588-589.

Strominger J. L., I 955. Uridine diphosphate Acetylglucosanine phosphate and Uridinediphosphate Acctylgalactosanine sulfate. Biochem. Biophys. Acta., 17, 283-285. 